Article

\title{
X-ray Pump-Probe Investigation of Charge and Dissociation Dynamics in Methyl Iodine Molecule
}

\author{
Li Fang ${ }^{1, *}$, Hui Xiong ${ }^{2}$, Edwin Kukk ${ }^{3}$ and Nora Berrah ${ }^{2, *}$ \\ 1 Center for High Energy Density Science, University of Texas, Austin, TX 78712, USA \\ 2 Department of Physics, University of Connecticut, Storrs, CT 06269, USA; alexionghui@hotmail.com \\ 3 Department of Physics and Astronomy, University of Turku, FI-20014 Turku, Finland; ekukk@utu.fi \\ * Correspondence: lifang@austin.utexas.edu (L.F.); nora.berrah@uconn.edu (N.B.); \\ Tel.: +1-512-471-3675 (L.F.); +1-860-486-0439 (N.B.)
}

Academic Editor: Kiyoshi Ueda

Received: 26 April 2017; Accepted: 15 May 2017; Published: 19 May 2017

\begin{abstract}
Molecular dynamics is of fundamental interest in natural science research. The capability of investigating molecular dynamics is one of the various motivations for ultrafast optics. We present our investigation of photoionization and nuclear dynamics in methyl iodine $\left(\mathrm{CH}_{3} \mathrm{I}\right)$ molecule with an X-ray pump X-ray probe scheme. The pump-probe experiment was realized with a two-mirror $\mathrm{X}$-ray split and delay apparatus. Time-of-flight mass spectra at various pump-probe delay times were recorded to obtain the time profile for the creation of high charge states via sequential ionization and for molecular dissociation. We observed high charge states of atomic iodine up to 29+, and visualized the evolution of creating these high atomic ion charge states, including their population suppression and enhancement as the arrival time of the second X-ray pulse was varied. We also show the evolution of the kinetics of the high charge states upon the timing of their creation during the ionization-dissociation coupled dynamics. We demonstrate the implementation of X-ray pump-probe methodology for investigating X-ray induced molecular dynamics with femtosecond temporal resolution. The results indicate the footprints of ionization that lead to high charge states, probing the long-range potential curves of the high charge states.
\end{abstract}

Keywords: free electron laser; X-ray pump-probe; molecular dynamics; charge dynamics

\section{Introduction}

Molecular dynamics, including nuclear motion and electronic motion, are one of the fundamental aspects in scientific investigations, the information of which provides microscopic insights into chemical reactions and biological processes. Photo-induced dynamics in molecules can be essential to natural processes as well as in applications: electron transportation fuels the photo-chemical energy transformation in photosynthesis [1], photoabsorption, and the subsequent molecular fragmentation leads to application in bioimaging [2] and radiation therapy [3]. Knowledge concerning the dynamics of the charge and the nuclei in a molecule, underlying phenomena-such as photoionization, charge migration, and molecular dissociation-underpins the potential for photo-control of these processes and therefore, the possibility for photo-steering chemical reactions at an atomic level, as well as for optimization of photo-production.

Due to the ultrafast nature of molecular dynamics, e.g., tens to a few hundred femtoseconds for nuclear motion and sub-femtosecond to a few femtoseconds for electronic motion, ultrafast optical tools are needed for the investigation of these dynamics. Tabletop optical lasers in near infrared, VUV, and XUV regimes have been widely applied in work regarding the evolution of nuclear wavepackets and electronic wavepackets, typically with a pump-probe scheme [4,5]. However, pump-probe experiments in the $\mathrm{X}$-ray regime with tabletop lasers are still a challenge, due to the low photon output 
in the X-ray photon range, e.g., from high harmonic generation [6]. With the recent developments in free electron lasers (FELs), short wavelength pump-probe experiments have become practical, due to the high flux of FELs and have been realized with various X-ray split and delay (XRSD) designs [7,8]. For accelerator-based X-ray sources, pulse splitting and delay can also be implemented in the electron accelerator stage, before creating the $\mathrm{X}$-ray pulses [9].

$\mathrm{X}$-ray photons can access specific atomic sites in a molecule by selective inner-shell ionization. This atomic-site specificity allows for creating localized charge and hence, tracing charge transfer even with a single pulse or with a pulse duration that is longer than the actual time scale of the dynamical process [10,11]. In a previous work [10], charger transfer from iodine to the methyl group in methyl iodine molecule $\left(\mathrm{CH}_{3} \mathrm{I}\right)$ was investigated using near-infrared (NIR) and FEL pulses. Therein, the INR pulse produced singly charged molecular ions and initiated the dissociation and the FEL pulse created positive charge localized at the iodine which transferred to the methyl group at a later time. In this work, the vanished atomic iodine ion population at short pump-probe delay time indicates the charge transfer from the iodine to the methyl group. Single FEL pulse was also used to investigate molecular dynamics in $\mathrm{CH}_{3} \mathrm{I}$ and methylselenol $\left(\mathrm{CH}_{3} \mathrm{SeH}\right)[11,12]$, where ion coincidence mapping was used for correlation of carbon and iodine charge states indicating the charge redistribution within the molecule. In single pulse experiments, the extraction of the timing information delay on theoretical models, typically involving Coulomb explosion [11,13].

In the present work, we used a two-mirror XRSD apparatus to generate two X-ray pulses for the pump-probe experiment where we investigated the molecular dynamics in $\mathrm{CH}_{3} \mathrm{I}$ molecule. Using a single-color X-ray pump and X-ray probe scheme, we were able to probe with an independent clock $X$-ray induced molecular dynamics, e.g., the sequential ionization and relaxation of highly excited molecular ionic states via fragmentation. Using time-of-flight mass spectroscopy, we obtained the time profile of high charge states of atomic iodine and the evolution map of the kinetic energy (KE) distribution for selected charge states. The results show suppression of high charge states at small pump-probe delay time, a dramatic increase till a delay time comparable to the pulse duration, and an enhancement at relatively longer delay times, all associated with modifications in charge state branching ratios. The KE distribution shows a monotonic width narrowing down as the pump-probe delay time increases, as a consequence of increasing abundance of slow ions.

\section{Materials and Methods}

The experiment was carried out at the atomic, molecular and optical physics (AMO) end station at the LCLS free electron laser facility (SLAC National Laboratory, Menlo Park, CA, USA). The photon energy used was $1619 \mathrm{eV}$ which dominantly ionizes the M1 subshell of iodine. The pulse duration for both pump and probe was $50 \mathrm{fs}$ and the pulse energy measured upstream the focusing mirrors and the XRSD was $1.3 \mathrm{~mJ}$. Considering an X-ray transportation loss of $85 \%$, the actual total pulse energy at the interaction region is estimated to be $\sim 0.2 \mathrm{~mJ}$, corresponding to an intensity of $4 \times 10^{16} \mathrm{~W} / \mathrm{cm}^{2}$ with a focal size of $\sim 10 \mu \mathrm{m}^{2}$. Gas phase $\mathrm{CH}_{3} \mathrm{I}$ was delivered using a pulsed valve jet. Ion Time of flight (TOF) signal was detected and recorded at different pump-probe delay times. The details of the experimental setup are described in [8].

The XRSD apparatus (see [8] for details) consists of two plane mirrors, is designed for soft X-ray photon energy range $(250 \mathrm{eV}-1800 \mathrm{eV})$ and is located downstream of the X-ray focusing mirrors of the AMO hutch. The positions (angles and height) of the two mirrors are adjusted by actuators to achieve the desired pump-probe delay time with a minimum time-resolution of 100 as and the maximum delay time of about $400 \mathrm{fs}$. The high position reproducibility of the actuators using position sensors make possible reliable pre-experiment calibration of the actuator settings for any particular pump-probe delay-time with optimal spatial overlap.

To extract the KE distribution of the ions, we first obtained with SIMION (v5, SIS Inc., Ringoes, NJ, USA) simulation the peak shapes B $\left(E_{j}, t_{i}\right)$ of the TOF response for a sequence of discrete 
energy release, $E_{j}(j=1,2, \ldots, n)$. These peak profiles are regarded as a set of base functions. The experimental TOF was fitted by the following function:

$$
\operatorname{TOF}\left(t_{i}\right)=\sum_{j=1}^{n} I_{j} B\left(E_{j}, t_{i}\right) \Delta E_{j}
$$

where $\Delta E_{j}$ is the energy interval and $I_{j}$ are the fitting coefficients.

We also used a point-charge Coulomb explosion model to calculate the time evolution for the various ionic charges. The model is described in detail in [11], where it was applied to a single-pulse scenario. The model is designed to represent, in average, the time evolution into any given set of final atomic fragment charges, including charge build-up, redistribution, and the concurrent Coulomb explosion. The sequence starts by single-photon absorption by the M1 subshell of iodine, with subsequent positive charge created by Auger cascades and possibly by additional photoabsorption. Due to large disparity in photoionization cross-sections in iodine and in the methyl group at this photon energy, only iodine is assumed to absorb the photons and charge build-up in the methyl group that occurs via electron dynamics, such as Auger cascades involving molecular orbitals. The statistical nature of the molecule is reflected in the smooth charge build-up function (instead of stepwise increase at random intervals for each single event)

$$
Q_{\text {tot }}(t)=Q_{\text {tot, final }}\left(1-\exp \left(-\frac{t}{\tau}\right)\right)
$$

where $\tau$ is the empirical, adjustable charge build-up constant.

\section{Results}

\subsection{Timeline for Generation of Highly Charged Atomic Iodine Ions}

With a photon energy of $1619 \mathrm{eV}$, the dominant ionization at the first ionization step by a single FEL pulse is the photoionization of the $\mathrm{M}_{1}$ shell electrons of the iodine atom which have a binding energy of $\sim 1072 \mathrm{eV}$. The subsequent cascade Auger decay and sequential decay will lead to high charge states of iodine. The second laser pulse, arriving at a particular delay time, will further ionize the molecular ions, likely dissociating after being generated by the first pulse. At short delay time, the ionization by the second pulse kicks in while the ionization by the first pulse is still ongoing. At longer delay, the second pulse will ionize established charge states during molecular dissociation.

We observed high charge states up to 29+ for iodine and 3+ for carbon, as shown in Figure 1a $\left(\mathrm{C}^{4+}\right.$ ion peaks marked in Figure 1a cannot be confirmed in the current experiment, because the estimated $\mathrm{KE}$ is $\sim 230 \mathrm{eV}$, an increase from that for $3+$ is much higher than expected or measured in previous work [11]). In the case of 'no overlap', we moved one of the two split mirrors to avoid spatial overlap of the two X-ray pulses to experimentally mimic the situation of single pulse with the same total pulse energy as two separate pulses. To ensure 'no overlap', we also set a long time delay of $150 \mathrm{fs}$. This single pulse condition will be presented in figures as the case of zero delay. The true time zero, the zero reference for all pump-probe delays, was set by mechanically making the two split mirrors co-plane [8]. With a single pulse ('no overlap'), the overall production of highly charged iodine ions is minimum even though the interaction region volume doubled, in comparison with the high charge state yield by two pulses. In contrast, the production of high charge states of carbon is at maximum in the single pulse scenario, which is similar to the production at long delay time in the two-pulse scenario. The spectral peaks for $\mathrm{I}^{21+}$ to $\mathrm{I}^{23+}$ overlap with those for $\mathrm{C}^{2+}$. Their contribution, however, broadened the $\mathrm{C}^{2+}$ peaks and even leads to a split structure on top of the fast $\mathrm{C}^{2+}$ peak at the delay time of $150 \mathrm{fs}$ (see Figure 1a). The $\mathrm{I}^{29+}$ peak also overlaps with a carbon peak, but is obviously seen on top of the slow $\mathrm{C}^{3+}$ peak. It is possible that charge states of iodine higher than $29+$ were produced but buried under the $\mathrm{C}^{3+}$ peak, as barely seen at the valley between the two $\mathrm{C}^{3+}$ peaks (see Figure 1a). 
Similarly, the $\mathrm{I}^{21+}$ appears between the two $\mathrm{C}^{2+}$ peaks. The abundance of high charge states decreases for higher charges. The seemingly higher yield of $\mathrm{I}^{16+}$ compared to the adjacent peaks is due to the contribution of $\mathrm{O}^{2+}$ from the background gas in the vacuum chamber. The production of low charge states $\left(\mathrm{I}^{+}\right.$to $\left.\mathrm{I}^{6+}\right)$ is favored at long delay time, reaching a maximum with a single pulse as shown in Figure $1 \mathrm{~b}$. At the low charge states, the ion yields at short ( $20 \mathrm{fs})$ and intermediate $(\sim 40 \mathrm{fs})$ delay times are similar.
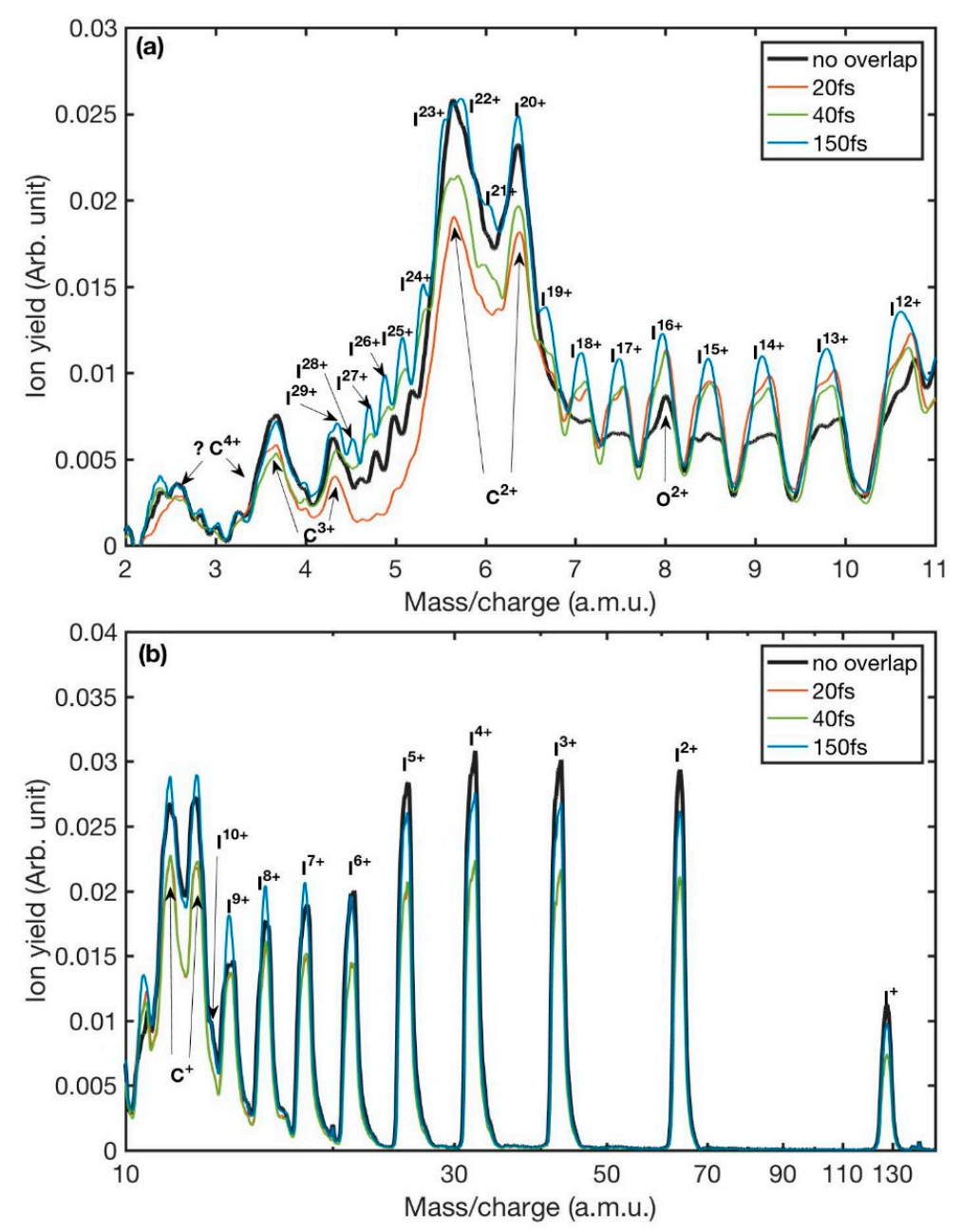

Figure 1. Ion Time of flight (TOF) spectra of $\mathrm{CH}_{3} \mathrm{I}$ at a photon energy of $1619 \mathrm{eV}$ and a pulse duration of $50 \mathrm{fs}$. Different curves (color coded) correspond to selected pump-probe delay times (see legend). The pulse energy is estimated to be $\sim 0.2 \mathrm{~mJ}$, based on $1.3 \mathrm{~mJ}$ upstream value and a transportation loss of $85 \%$. 'no overlap' is considered to represent the case at zero delay time (see text). (a) Spectral range: $<11 \mathrm{amu}$; (b) Spectral range: $>10 \mathrm{amu}$. the $x$-axis is plotted in log scale.

Figure 2 shows the TOF spectra as a function of pump-probe delay with a finer step size (see figure $y$-axis tick labels). The time zero spectrum corresponds to that with single pulse mentioned above. As seen in Figure 2, there is an overall drop in the ionization yield at short pump-probe delay time (20 fs). Particularly, the very high charge states $(>24+)$ are almost vanished. $\mathrm{Most} \mathrm{CH}_{3} \mathrm{I}$ molecular ions went through dissociation, since only a small amount of $\mathrm{CH}_{3} \mathrm{I}^{+}$remains (see Figure $1 \mathrm{~b}$ ). The first significant peak is $\mathrm{I}^{+}$, which is much smaller compared to the dominant $\mathrm{I}^{2+}$ peak, because Auger decay preferentially generates evenly charged ions and the charges are mainly localized. As the pump-probe delay time increases, the ion yield for high charge states increases within the pulse duration. The very high charge state $(>24+)$ peaks reached their maxima at $\sim 100 \mathrm{fs}$ time delay, meanwhile the abundance of mid and low high charge states increases (see Figure 2). In Figure 2a, one clearly sees the rising 
peaks of $\mathrm{I}^{19+}, \mathrm{I}^{21+}$, and $\mathrm{I}^{24+}$ above the shoulders of the dominant $\mathrm{C}^{2+}$ peaks. Also, becoming clear are the peaks of $\mathrm{I}^{22+}$ and $\mathrm{I}^{23+}$ which appear as a split branch on top of the fast $\mathrm{C}^{2+}$ peak.

Charge states of carbon ions up to $3+$ were observed. The highest charge state reached does not depend on the delay time. As seen in Figure $1, \mathrm{C}^{3+}$ was produced with a single pulse or at short delay time. However, the abundance of these carbon ions shows a delay-time dependence, increasing for longer delay time. The contributions of iodine ions under the carbon peaks are not large enough to explain the changes in the carbon peaks which are dominant. With a single pulse, the carbon ion signal at all charge states reaches around the maxima, similar to the values at long delay time. This could be an indication of charge transfer from the iodine to the methyl group that was thwarted by the ionization with the probe pulse [10].

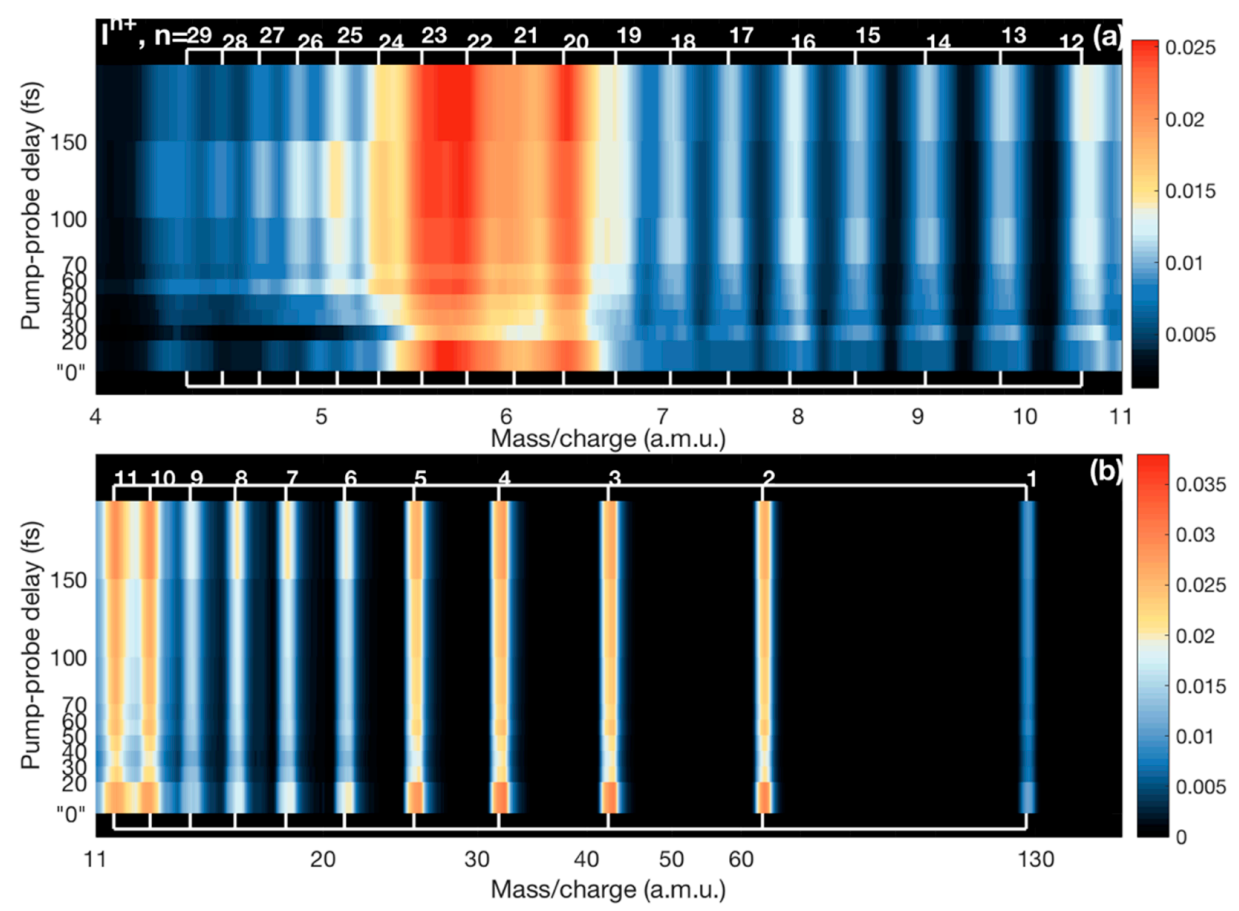

Figure 2. Ion TOF spectra as a function of pump-probe delay time at a photon energy of $1619 \mathrm{eV}$ and a pulse duration of $50 \mathrm{fs}$. The color represents the ion signal per laser shot (arb. unit) (see color bar). The " 0 " delay corresponds to the case of no overlap between the two X-ray beams (see text). The positons for various charge states of iodine atomic ions are marked and labeled at the top of the figure. (a) Spectral range: mass/charge < 11 amu; (b) Spectral range: mass/charge $>10$ amu. The $x$-axes are plotted in log scale.

To illustrate the overall picture of the evolution of the iodine charge states, we integrated the signal under the spectral peaks of various charge states at different pump-probe times, as shown in Figure 3. The integral signal with a single pulse was subtracted from the integrated signal at all other delay times, i.e., using the single pulse spectrum as a reference. Clearly seen in Figure 3 is: (1) high charge states were enhanced and low charge states were partially depleted by the second FEL pulse, shown as the trend from negative to positive ion yield difference for increasing charge states; (2) at short delay time, the depletion of low charge states were stronger compared to that at long delay, shown as the trend of decreasing absolute ion yield difference for increasing delay time within the block for each different charge state; (3) long delay time favors the production of very high charge states, consistent with the theme that these charge states were suppressed at $20 \mathrm{fs}$ delay (negative values for $\mathrm{I}^{25+}$ to $\mathrm{I}^{28+}$ ); (4) $50 \mathrm{fs}$ delay, which is equal to the pulse duration, is an odd point at which the low and very high charges are dramatically enhanced, but not the intermediate charge states, compared to the case at adjacent delay times. 


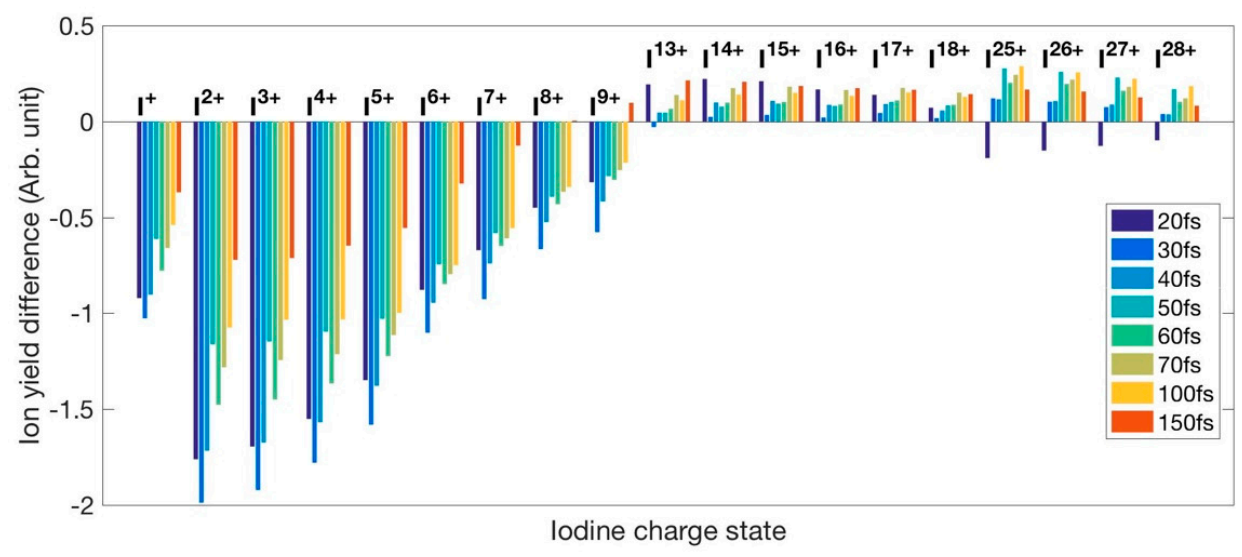

Figure 3. Ion yield as a function of pump-probe delay time for various iodine charge states. Data were extracted from TOF spectra in Figure 2. The ion yield with a single X-ray pulse was used as a reference spectrum and subtracted from the iodine charge states at all other delay times.

As roughly seen in Figure 2a, there seems to be a phase shift between the spectrum with single pulse and those at delays larger than $50 \mathrm{fs}$ for charge states $\mathrm{I}^{25+}$ to $\mathrm{I}^{28+}$. The spectra of the same charge state region are shown in Figure 4, where a decay base line of the spectrum at 20 fs delay was subtracted from all spectra and the spectrum at $20 \mathrm{fs}$ delay was amplified for clarity of comparison. The 'phase shift' actually reflects the fact that at single pulse or small delay times, ions with large KE were produced; as the delay time increases, low KE ions were produced, filling the valley between the forward and backward peaks of the high KE ion and even forming a new peak for large delay time. At intermediate delay times, around $30 \mathrm{fs}$, the transient case is shown as multiple peaks from both above origins are seen.

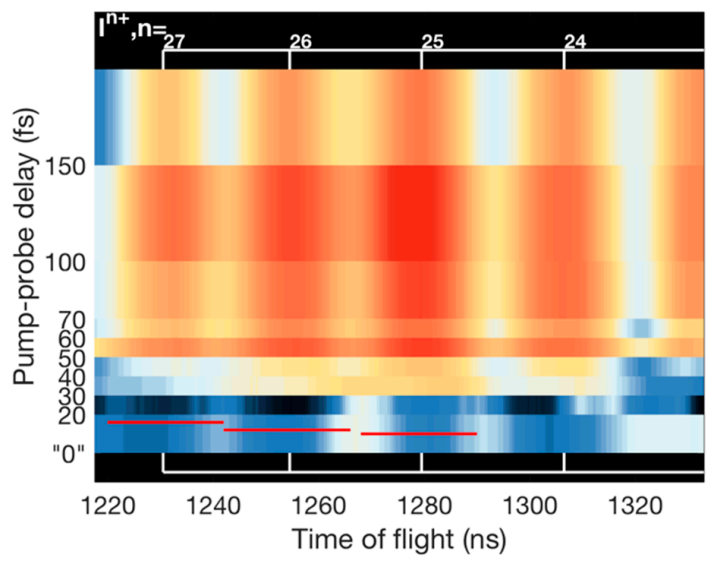

Figure 4. Ion TOF spectra as a function of pump-probe delay time. The data were from Figure 2a with the $x$-axis plotted in TOF. A background trace obtained for the spectrum at $20 \mathrm{fs}$ was subtracted from all spectra. The spectrum at $20 \mathrm{fs}$ delay was magnified by $\times 6$ for purpose of clarity for presentation. The ' 0 ' delay corresponds to the case of no overlap between the two X-ray beams (see text). At the top of the figure, the positions for the charge states of atomic iodine were marked and labeled. The red horizontal lines indicate the TOF spread of the forward and backward peaks of $\mathrm{I}^{25+}, \mathrm{I}^{26+}$, and $\mathrm{I}^{27+}$, identified in the spectrum at $20 \mathrm{fs}$ or single pulse.

\subsection{Evolution of KE Distribution}

As seen in Figure 1a, with a single pulse, the KE for high charge states $\left(\mathrm{I}^{12+}-\mathrm{I}^{18+}\right)$ has a broad distribution, shown as a flat top shape in the TOF spectrum. As the delay time increases, the abundance of ions with small KE increased, adding signal on top of the flat top, eventually leading to a sharp dome 
shape, as seen at long delay times. As to extremely high charge states of iodine $\left(\mathrm{I}^{20+}-\mathrm{I}^{29+}\right)$, a sharp distribution starts at short delay time. For low charge states (shown in Figure 1b), the distribution is relatively sharp even at single pulse or short delay time, indicating small average KEs. Similarly seen in Figure 2a, the broad KE distribution with a single pulse is represented by an evenly colored rectangular region at around zero delay time. As more low KE ions were detected at longer delay times, the shape of distribution becomes sharper, forming slim columns (see Figure 2).

We extract the ion KE from the TOF spectra with the method described in Section 2. Figure 5 shows the KE of $\mathrm{I}^{14+}$ as a function of pump-probe delay time. Each KE spectrum was normalized to its maximum. The map was interpreted without significant distortion of the distribution to smooth out the uneven delay-time steps. The ion species of $\mathrm{I}^{14+}$ was selected as an example to show the evolution of the KE distribution and other high charge state species show a similar trend (see Figure 1). The difference between the distribution obtained from the forward ions and backward ions is mainly due to the uneven base line caused by spectral overlap with the adjacent peaks. Figure 6 shows the weighted KE averaged over the forward and backward ions. At small delay time, the KE of $\mathrm{I}^{14+}$ shows a broad distribution with an average $\mathrm{KE}$ of $10 \mathrm{eV}$ and a width of $\sim 15 \mathrm{eV}$. At about $60 \mathrm{fs}$ delay time, the KE distribution approaches an asymptotic value of $\sim 8 \mathrm{eV}$ with a width of $\sim 8 \mathrm{eV}$.
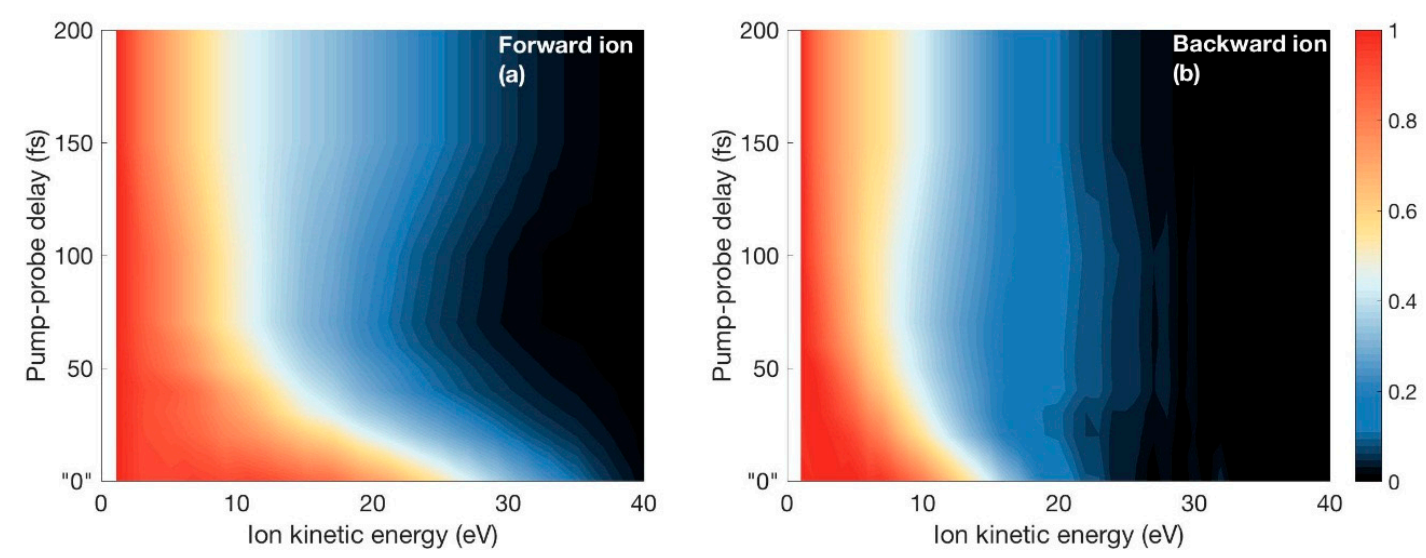

Figure 5. Ion KE distribution of $\mathrm{I}^{14+}$ as a function of pump-probe delay time at a photon energy of $1619 \mathrm{eV}$ and a pulse duration of $50 \mathrm{fs}$. The color represents the ion signal strength which is normalized to the signal maximum at each delay time. The ' 0 ' delay corresponds to the case of no overlap between the two X-ray beams (see text). (a) Ions with the direction of the initial velocity towards the detector ('forward ion'); (b) Ions with the direction of the initial velocity against the detector ('backward ion').

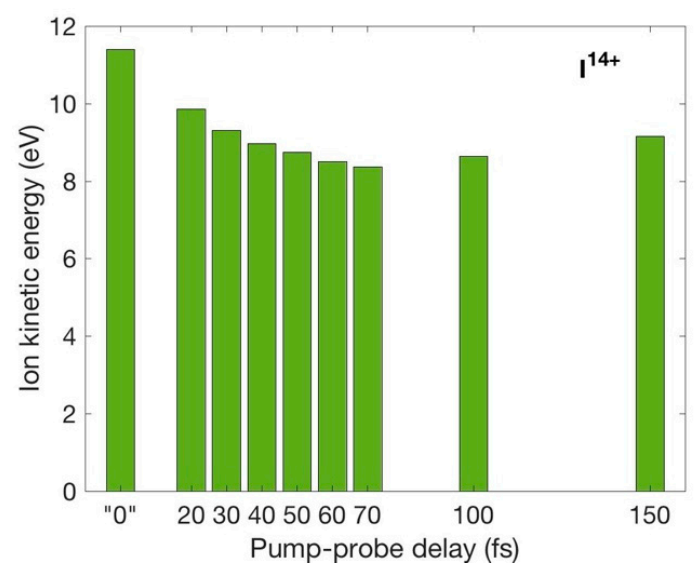

Figure 6. Weighted KE averaged over the forward and backward $\mathrm{I}^{14+}$ ions as a function of pump-probe delay time, extracted from data shown in Figure 5 . The ' 0 ' delay corresponds to the case of no overlap between the two X-ray beams (see text). 


\section{Discussion}

As mentioned above, two pulses (with spatial overlap) produced more high charge state iodine ions than a single pulse even with the same total pulse energy. The reason is that the ions generated by the first pulse and further ionized by the second pulse are associated with higher ionization potentials which are closer to the photon energy and therefore have larger ionization cross sections compared to the neutral particles. Since the life time of the iodine M1 shell is only 435 as [14], much shorter than the X-ray pulse duration, the suppression of every high charge states at short delay time is likely due to the depletion of valence shell electrons which hinders the Auger decay, rather than X-ray induced photoabsorption frustration caused by inner-shell electron depletion which hinders sequential photoionization [15,16]. Indeed, with a photon energy of $1916 \mathrm{eV}$, photoionization of M1 shell electron alone can only lead to up to $\mathrm{I}^{26+}$, since the binding energy of a 3 s electron of $\mathrm{I}^{26+}$ (with all valence vacancies) is $\sim 1680 \mathrm{eV} \mathrm{[17].} \mathrm{At} \mathrm{long} \mathrm{delay} \mathrm{times,} \mathrm{the} \mathrm{population} \mathrm{in} \mathrm{iodine} \mathrm{high} \mathrm{charge} \mathrm{states} \mathrm{increased,}$ because the long delay times allow for the completion of the electron transfer from carbon sites to the methyl group, restocking the valence electrons at iodine needed for the Auger decay.

We obtained the average charge of the atomic iodine ions for different pump-probe delay times, as shown in Figure $7 \mathrm{a}$. With a single FEL pulse, the average charge of iodine is $\sim 7 \mathrm{amu}$; with the second pulse, a charge of $\sim 9$ amu was established around $60 \mathrm{fs}$. The experimental results show a charge saturation at $\sim 30 \mathrm{fs}$. We simulated the charge evolution in a pump-probe experiment, as shown in Figure $7 \mathrm{~b}$. The charge by a single X-ray pulse was set to be $\sim 7 \mathrm{amu}$ and a charge built up time of $\sim 30 \mathrm{fs}$ was used. Since the photoionization cross section for carbon is more than one order of magnitude less than that of iodine at $1619 \mathrm{eV}$, photoionization of carbon was excluded in the simulation. Hence, the carbon charge mainly resulted from the charge transfer between the iodine and the methyl group, which can charge the carbon site up to $4+[10,11]$. As seen in Figure $7 \mathrm{~b}$, the charge on carbon is not affected by the probe pulse arriving $50 \mathrm{fs}$ later after the pump pulse, because the charge transfer was hindered due to the large C-I bond separation at the latter time of the molecular dissociation.

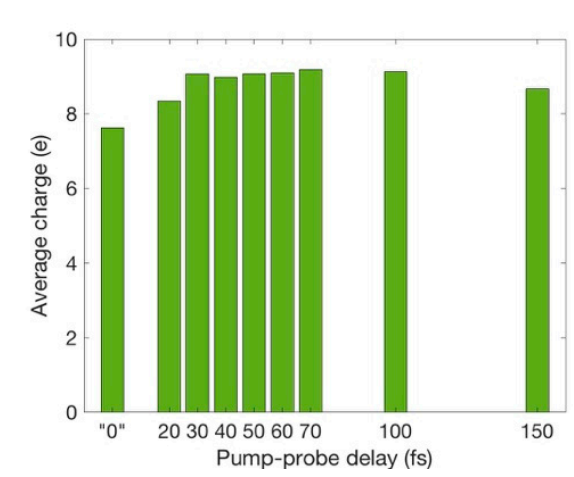

(a)

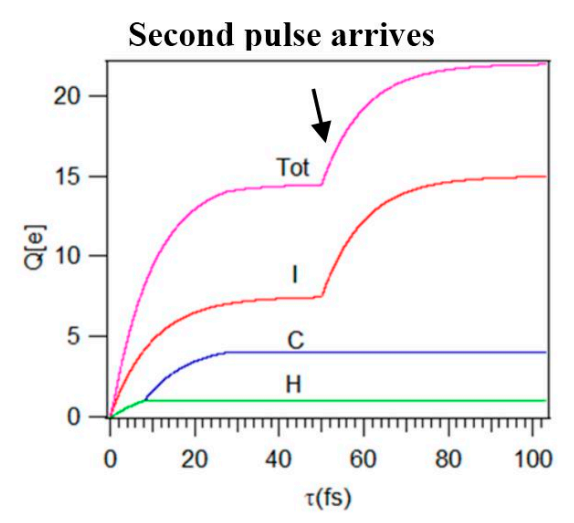

(b)

Figure 7. (a) Experimental average charge of iodine as a function of pump-probe delay time. The ' 0 ' delay corresponds to the case of no overlap between the two X-ray beams (see text); (b) Simulation of charges as a function of time for different ionic species. Pulse duration of $50 \mathrm{fs}$ and delay time of $50 \mathrm{fs}$ were used. The $x$-axis is time with zero position at the onset of the first pulse.

From the TOF spectral peaks with single pulse, we estimated the KEs for $\mathrm{I}^{25+}, \mathrm{I}^{26}$, and $\mathrm{I}^{27+}$ (corresponding to horizontal lines in Figure 4, see figure caption), as well as the KEs for the carbon ions and their associated iodine ions on average. The results are presented in Table 1. Also shown in Table 1 for comparison are the KEs of carbon ions estimated from the results reported in [11]. Since the C-I bond breaks within the first 10 fs of the X-ray pulse [11], we expect the $\mathrm{KE}$ for $\mathrm{C}^{+}$which was dominantly produced at an early time during the FEL pulse to be similar between the experiments in current work and in [11]. This is indeed the case as shown in Table 1. However, for higher carbon charge states 
$(2+, 3+)$, the KEs in the current work are much lower than those in [11]. A plausible explanation is: the high charge states observed in [11] were produced within $10 \mathrm{fs}$ of a single pulse, where one can consider a scenario of internal clock pump-probe experiment $[13,18]$; at the very short effective delay time, the dissociation at short range of the internuclear separation was probed, leading to large KEs due to stronger Coulomb repulsion; whereas in the current work where an external independent clock was applied, allowing for relatively larger delay times, the potential curve at long range was probed, leading to lower dissociation energy at the end. As an example, for low charge states of iodine, we estimated the KE for $\mathrm{I}^{4+}$ to be $4.5 \mathrm{eV}$ which is nearly constant for different delay times, indicating that the $\mathrm{C}^{3+}$ ions are likely to be associated with $\mathrm{I}^{4+}$. With a similar reasoning, the very high charge states of iodine are likely associated with high charge states of carbon, but due to their small abundance, the contribution of the associated carbon ions is also small.

Table 1. Kinetic energy (KE) of carbon and iodine ions. The values for the current work were obtained from the spectrum with single pulse. The charge states in the parenthesis are the species associated those for which the KEs are presented.

\begin{tabular}{|c|c|c|c|c|c|c|c|}
\hline Species & $\mathrm{KE}(\mathrm{eV})^{1}$ & Species & $\mathrm{KE}(\mathrm{eV})^{1}$ & Species & $\mathrm{KE}(\mathrm{eV})^{1}$ & Species & $\mathrm{KE}(\mathrm{eV})^{2}$ \\
\hline $\mathrm{C}^{+}\left(-\mathrm{I}^{\mathrm{n}+}\right)$ & $5.0 \pm 0.9$ & $\mathrm{I}^{\mathrm{n}+}\left(-\mathrm{C}^{+}\right)$ & $0.5 \pm 0.3$ & $\mathrm{I}^{25+}$ & $49 \pm 9$ & $\mathrm{C}^{+}\left(-\mathrm{I}^{+}\right)$ & $\sim 6$ \\
\hline $\mathrm{C}^{2+}\left(-\mathrm{I}^{\mathrm{n}+}\right)$ & $19.1 \pm 2.3$ & $\mathrm{I}^{\mathrm{n}+}\left(-\mathrm{C}^{2+}\right)$ & $1.8 \pm 0.2$ & $\mathrm{I}^{26+}$ & $61 \pm 10$ & $\mathrm{C}^{2+}\left(-\mathrm{I}^{5+}\right)$ & $\sim 47$ \\
\hline $\mathrm{C}^{3+}\left(-\mathrm{I}^{\mathrm{n}+}\right)$ & $42.5 \pm 7.9$ & $\mathrm{I}^{\mathrm{n}+}\left(-\mathrm{C}^{3+}\right)$ & $4.0 \pm 0.4$ & $\mathrm{I}^{27+}$ & $55 \pm 10$ & $\mathrm{C}^{3+}\left(-\mathrm{I}^{7+}\right)$ & $\sim 100$ \\
\hline
\end{tabular}

${ }^{1}$ Current work. The KEs of $\mathrm{I}^{\mathrm{n}+}$ are extracted from that of $\mathrm{C}$ ion, assuming a two-body break-up. ${ }^{2}$ Reference [11]. Single pulse experiment with FEL pulses of $10 \mathrm{fs}$ duration and $5.5 \mathrm{keV}$ photon energy.

As mentioned above, the KE approaches a constant value at $~ 60$ fs delay and the ion yield at $50 \mathrm{fs}$ delay is an odd point in the overall trend. It remains a question whether this tip point around $50 \mathrm{fs}$ for the kinetics is a pulse duration factor or a species-dependent factor. Seeking the answer will be for future investigations with different FEL pulse durations. The pulse duration was not directly measured but derived based on electron bunch duration measurements [15]. The actual pulse duration has been reported to be about half of the cited value [19]. Considering an actual pulse duration of $25 \mathrm{fs}$ for a nominal value of $50 \mathrm{fs}$, the interference between the pump and probe pulses is not expected to be an issue in the current work.

\section{Conclusions}

We presented an investigation of the ionization and dissociation dynamics in $\mathrm{CH}_{3} \mathrm{I}$ molecule with an X-ray pump and X-ray probe scheme. To illustrate the charge dynamics and nuclear kinetics, we showed the charge state and the KE distribution for selected charge states as a function of pump-probe delay time. We observed the creation of ions with low $\mathrm{KE}$ at a relatively long delay time, probing the long-range potential curves of molecular high charge states. We also observed the suppression of high charge states at short delay time and an increase of the high charge states at long delay time, indicating the time scale of the C-I charge transfer. X-ray pump-probe experimental approach combined with coincidence measurements for resolving fragmentation channels could hold the promise for unambiguous time-resolved investigation of molecular dynamics, including decoupled dissociation and charge transfer dynamics.

Acknowledgments: We acknowledge the XRSD commissioning team: Brendan F. Murphy, Timur Y. Osipov, Ryan Coffee, Ken R. Ferguson, Jean-Charles Castagna, Vladmire S. Petrovic, Sebastian Carron Montero, and John D. Bozek. This work was funded by the Department of Energy, Office of Science, Basic Energy Sciences (BES), Division of Chemical Sciences, Geosciences, and Biosciences under a SISGR program1 No. DE-SC0002004, under grant No. DE-SC0012376 and for the Linac Coherent Light Source (LCLS), SLAC National Accelerator Laboratory, under Contract No. DE-AC02-76SF00515. E.K. acknowledges the support by the Academy of Finland. L.F. acknowledges the support by Defense Advanced Research Project Agency Contract 12-63-PULSE-FP014, and by National Nuclear Security Administration Cooperative Agreement DE-NA0002008. 
Author Contributions: N.B. conceived the experiment; L.F., H.X., E.K. and N.B. performed the experiments; H.X. and L.F. analyzed the data; E.K. did the simulation; L.F. wrote the paper.

Conflicts of Interest: The authors declare no conflict of interest.

\section{References}

1. Lewis, K.L.M.; Ogilvie, J.P. Probing photosynthetic energy and charge transfer with two-dimensional electronic spectroscopy. J. Phys. Chem. Lett. 2012, 3, 503-510. [CrossRef] [PubMed]

2. Neustetter, M.; Aysina, J.; da Silva, F.F.; Denifl, S. The Effect of Solvation on Electron Attachment to Pure and Hydrated Pyrimidine Clusters. Angew. Chem. Int. Ed. Engl. 2015, 54, 9124-9126. [CrossRef] [PubMed]

3. Khan, F.M. Part I 5. Interactions of Ionizing radiation. In The Physics of Radiation Therapy, 3rd ed.; Lippincott Williams\&Wilkins: Philadelphia, PA, USA, 2003.

4. Magrakvelidze, M.; Herrwerth, O.; Jiang, Y.H.; Rudenko, A.; Kurka, M.; Foucar, L.; Kühnel, K.U.; Kübel, M.; Johnson, N.G.; Schröter, C.D.; et al. Tracing nuclear-wave-packet dynamics in singly and doubly charged states of $\mathrm{N}_{2}$ and $\mathrm{O}_{2}$ with XUV-pump-XUV-probe experiments. Phys. Rev. A 2012, 86, 013415. [CrossRef]

5. Leone, S.R.; McCurdy, C.W.; Burgdörfer, J.; Cederbaum, L.S.; Chang, Z.; Dudovich, N.; Feist, J.; Greene, C.H.; Ivanov, M.; Kienberger, R.; et al. What will it take to observe processes in 'real time'? Nat. Photonics 2014, 8, 162-166. [CrossRef]

6. Hädrich, S.; Rothhardt, J.; Krebs, M.; Demmler, S.; Klenke, A.; Tünnermann, A.; Limpert, J. Single-pass high harmonic generation at high repetition rate and photon flux. J. Phys. B At. Mol. Opt. Phys. 2016, 49, 1-26. [CrossRef]

7. Woestmann, M.; Mitzner, R.; Noll, T.; Roling, S.; Siemer, B.; Siewert, F.; Eppenhoff, S.; Wahlert, F.; Zacharias, H. The XUV split-and-delay unit at beamline BL2 at FLASH. J. Phys. B At. Mol. Opt. Phys. 2013, 46, 164005. [CrossRef]

8. $\quad$ Berrah, N.; Fang, L.; Murphy, B.F.; Kukk, E.; Osipov, T.Y.; Coffee, R.; Ferguson, K.R.; Xiong, H.; Castagna, J.C.; Petrovic, V.S.; et al. Two mirror X-ray pulse split and delay instrument for femtosecond time resolved investigations at the LCLS free electron laser facility. Opt. Express 2016, 24, 11768-11781. [CrossRef] [PubMed]

9. Ding, Y.; Decker, F.J.; Emma, P.; Feng, C.; Field, C.; Frisch, J.; Huang, Z.; Krzywinski, J.; Loos, H.; Welch, J.; et al. Femtosecond X-ray pulse characterization in free-electron lasers using a cross-correlation technique. Phys. Rev. Lett. 2012, 109, 254802. [CrossRef] [PubMed]

10. Erk, B.; Boll, R.; Trippel, S.; Anielski, D.; Foucar, L.; Rudek, B.; Epp, S.W.; Coffee, R.; Carron, S.; Schorb, S.; et al. Imaging charge transfer in iodomethane upon X-ray photoabsorption. Science 2014, 345, 288-291. [CrossRef] [PubMed]

11. Motomura, K.; Kukk, E.; Fukuzawa, H.; Wada, S.; Nagaya, K.; Ohmura, S.; Mondal, S.; Tachibana, T.; Ito, Y.; Koga, R.; et al. Charge and Nuclear Dynamics Induced by Deep Inner-Shell Multiphoton Ionization of $\mathrm{CH}_{3} \mathrm{I}$ Molecules by Intense X-ray Free-Electron Laser Pulses. J. Phys. Chem. Lett. 2015, 6, 2944-2949. [CrossRef] [PubMed]

12. Erk, B.; Rolles, D.; Foucar, L.; Rudek, B.; Epp, S.W.; Cryle, M.; Bostedt, C.; Schorb, S.; Bozek, J.; Rouzee, A.; et al. Ultrafast Charge Rearrangement and Nuclear Dynamics upon Inner-Shell Multiple Ionization of Small Polyatomic Molecules. Phys. Rev. Lett. 2013, 110, 053003. [CrossRef] [PubMed]

13. Fang, L.; Osipov, T.; Murphy, B.; Tarantelli, F.; Kukk, E.; Cryan, J.P.; Glownia, M.; Bucksbaum, P.H.; Coffee, R.N.; Chen, M.; et al. Multiphoton ionization as a clock to reveal molecular dynamics with intense short X-FEL pulses. Phys. Rev. Lett. 2012, 109, 263001. [CrossRef] [PubMed]

14. Dampbell, J.L.; Papp, T. Widths of the atomic K-N7 levels. Atom. Data Nucl. Data 2001, 77, 1-56. [CrossRef]

15. Young, L.; Kanter, E.P.; Krässig, B.; Li, Y.; March, A.M.; Pratt, S.T.; Santra, R.; Southworth, S.H.; Rohringer, N.; DiMauro, L.F.; et al. Femtosecond Electronic Response of Atoms to Ultra-Intense X-rays. Nature 2010, 466, 56-61. [CrossRef] [PubMed]

16. Hoener, M.; Fang, L.; Kornilov, O.; Gessner, O.; Pratt, S.T.; Gühr, M.; Kanter, E.P.; Blaga, C.; Bostedt, C.; Bozek, J.D.; et al. Ultraintense X-ray Induced Ionization, Dissociation, and Frustrated Absorption in Molecular Nitrogen. Phys. Rev. Lett. 2010, 104, 253002. [CrossRef] [PubMed]

17. Estimated Using Los Alamos Atomic Physics Codes. Available online: http://aphysics2.lanl.gov/cgi-bin/ ION/runlanl08f.pl (accessed on 26 April 2017). 
18. Fang, L.; Osipov, T.; Murphy, B.F.; Rudenko, A.; Rolles, D.; Petrovic, V.; Bostedt, C.; Bozek, J.D.; Bucksbaum, P.H.; Berrah, N. Probing ultrafast electronic and molecular dynamics with free electron lasers. J. Phys. B At. Mol. Opt. Phys. 2014, 47, 124006. [CrossRef]

19. Murphy, B.F.; Osipov, T.; Jurek, Z.; Fang, L.; Son, S.K.; Mucke, M.; Eland, J.H.D.; Zhaunerchyk, V.; Feifel, R.; Avaldi, L.; et al. Bucky ball explosion by intense femtosecond X-ray pulses: A model system for complex molecules. Nat. Commun. 2014, 5, 4281. [CrossRef] [PubMed]

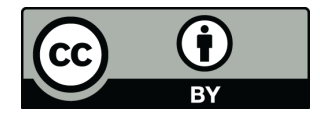

(C) 2017 by the authors. Licensee MDPI, Basel, Switzerland. This article is an open access article distributed under the terms and conditions of the Creative Commons Attribution (CC BY) license (http:/ / creativecommons.org/licenses/by/4.0/). 